Background and Aims Premature neonates commonly receive red blood cell (RBC) transfusions. Our aim was to systematically review the randomised controlled trial (RCT) evidence for use of RBC transfusions.

Methods We identified RCTs where the intervention was 'transfusion of red blood cells' from searches of multiple databases. Two reviewers independently extracted data and assigned overall quality. The primary review outcomes were mortality, and neurodevelopmental and respiratory endpoints.

Results We identified 27 RCTs; three studies compared RBC transfusion versus no transfusion/placebo, four compared transfusions of differing doses or administration schedule, 14 compared different types or products of RBC and six compared different thresholds for transfusion. Within the group of product trials, the largest subgroup of seven RCTs evaluated different media for storage or dilution of red cells, enrolling a total of 221 neonates. In the threshold group of six trials, enrolling 679 neonates, no significant differences in mortality (RR 1.22, 95\% C.I 0.84-1.75) or chronic lung disease (RR 0.99, 95\% C.I 0.84-1.15) were found; Only one RCT assessed neurodevelopment at 2 years and reported no difference. Many trials failed to report on clinical outcomes including mortality, chronic lung disease or other major neonatal co-morbidities, which would be considered of importance to clinicians.

Conclusions There are a large number of RCTs of RBC transfusions in this high risk population. Despite this, areas of concern included the nature of the intervention, outcome measures, sample size and quality of the trials, which precluded clear recommendations on the safety and role of RBC transfusion.

\section{FETAL HAEMOGLOBIN LEVELS IN PRETERM INFANTS AT 36 WEEKS POSTMENSTRUAL AGE: EFFECT OF BRONCHOPULMONARY DYSPLASIA, SEPSIS AND TRANSFUSIONS}

doi:10.1136/archdischild-2012-302724.0258

'B Perrone, 2P Marchionni, 'A Bartoli, 'C Gidiucci, 'M Pasqualini, 'R D'Ascenzo, 'F Marinelli, 'C Cameli, 3PE Cogo, 'VP Carnielli. 'Division of Neonatology; ${ }^{2} \mathrm{DIISM}$, Polytechnic University of Marche, Ancona; ${ }^{3}$ Pediatric Cardiac Anesthesia/Intensive Care Unit, Department of Pediatric Cardiology and Cardiac Surgery, Bambino Gesù Children's Hospital, Rome, Italy

Background and Aims Previous studies showed that switchover from fetal $(\mathrm{HbF})$ to adult $(\mathrm{HbA})$ haemoglobin occurs in relation to postmenstrual age (PMA).

Aims To assess $\mathrm{HbF}$ levels at 36 weeks PMA in preterm infants born between 24 and 31 completed weeks, to determine their association with bronchopulmonary dysplasia (BPD), sepsis and packedred-blood-cells (PRBC) transfusions.

Methods A retrospective cohort study of 130 preterm infants was performed.HbF determinations were obtained from the routine capillary blood gasses using ABL-800-flex (Radiometer Copenhagen) and results were reported as percentage of total $\mathrm{Hb}$. Associations of $\mathrm{HbF}$ levels and clinical variables were tested by t-test and multiple regression analysis.

Results Infants were born at a mean gestational age of 28.1 weeks (range 24-31.5 weeks), with a mean birth weight of $995 \mathrm{~g}$ (range 380-1965 g); 45 of them (34.6\%) had BPD, 36 (27.7\%) were affected by sepsis and $76(58.5 \%)$ received PRBC-transfusions (mean transfusion rate 2.5). At the univariate analysis $\mathrm{HbF}$ was significantly lower in infants with BPD $(54.3 \% \pm 21.2 \%$ vs $62.9 \% \pm 18 \% ; p=0.03)$, in those with sepsis $(49 \% \pm 18.7 \%$ vs $63 \% \pm 18.6 \%$; $=0.002)$ and in infants who received PRBC-transfusions $(48.8 \% \pm 14.6 \%$ vs $74.6 \% \pm 17.2 \%$; $\mathrm{p}<0,001)$. By multiple regression analysis, lower $\mathrm{HbF}$ levels were significantly associated to greater number of transfusions $(p<0.001)$, previous occurrence of sepsis $(\mathrm{p}=0.01)$ and BPD $(\mathrm{p}=0.05)$.
Conclusions PRBC-transfusions, sepsis and BPD are associated with lower $\mathrm{HbF}$ levels at 36 weeks PMA. Information on postnatal changes of $\mathrm{HbF}$ level and its related factors will help better understanding of oxygen transport in selected complications of prematurity.

\section{FROZEN PLASMA AND CRYOPRECIPITATE TRANSFUSION PRACTICES AMONG NEONATOLOGISTS - A NATIONAL SURVEY}

doi:10.1136/archdischild-2012-302724.0259

${ }^{1}$ RA Khan, ${ }^{1} V$ Venkatesh, ${ }^{1} \mathrm{~A}$ Curley, ${ }^{2} \mathrm{~S}$ Stanworth, ${ }^{3} \mathrm{C}$ Josephson, ${ }^{4} \mathrm{~B}$ Potterjoy, PlaNeT 2 Study Group. 'Neonatology, Cambridge University Hospitals NHS Foundation Trust; ${ }^{2}$ Haematology, John Radcliffe Hospital, Cambridge, UK; ${ }^{3}$ Paediatrics \& Pathology, Emroy University School of Medicine Atlanta, Atlanta, GA; ${ }^{4}$ Neonatology, Drexel University College of Medicine, Philadelphia, PA, USA

Background Frozen Plasma (FP) and cryoprecipitate (Cryo) are frequently transfused to neonates although indications remain unclear. This survey aimed to characterize current UK neonatal FFP/Cryo transfusion practices.

Methods Pre-piloted 15 question survey developed by neonatologists and transfusion medicine specialists in UK, to include clinical scenarios and direct questions about FP/Cryo transfusion decisions. Survey was posted to all neonatal units ( $n=200$ UK).

Results Response rate for UK was 53\%, 42\% Level 2, 25\% level 3 and $12 \%$ level 1 units. $48 \%$ of clinicians, would consider using FP for volume expansion in a non-bleeding, non-coagulopathic infant with hypotension refractory to inotropes and crystalloids. In a clinical vignette describing the same case scenario, $11 \%$ of clinicians would use FP to raise oncotic pressure. 19\% of neonatologists would use FP as an adjunct to diuresis in an infant with oedema, when other interventions failed, 5\% of clinicians would order FP. For isolated abnormal haemostatic test results in the absence of bleeding, $21 \%$ of clinicians would give FP (irrespective of laboratory results), while $66 \%$ would order FP for clinical bleeding in the absence of coagulation results. When asked about volume of FP administered, $51 \%$ responded $10 \mathrm{mls} / \mathrm{kg}$, and $43 \% 15-20 \mathrm{mls} / \mathrm{kg}$. With respect to cryoprecipitate, $26 \%$ of respondents did not use this product.

Conclusions FP practice including dose is highly variable. Neonatologists are considering use of FP to expand volume and raise oncotic pressure. There is limited consideration of Cryo for transfusion. This survey highlights areas where evidence and education are essential to improve practice.

\section{A SINGLE CENTRE RETROSPECTIVE STUDY OF CYTOGENETIC PROFILE, MANAGEMENT AND OUTCOME OF ACUTE MYELOID LEUKAEMIA IN CHILDREN}

doi:10.1136/archdischild-2012-302724.0260

P O'Hare, M Taj, D Lancaster. Paediatric Oncology, Royal Marsden Hospital, London, UK

Background and Aims AML15 was the first major trial to compare anthracycline based consolidation to a Cytosine-Arabinoside based one. Following closure of the trial, standard therapy of cytosine - arabinoside consolidation therapy is currently recommended at this centre. A review of the cytogenetic, treatment and outcome profiles for patients diagnosed with Acute Myeloid Leukaemia was undertaken following concerns regarding relapse rates.

Methods A retrospective study was conducted at the Royal Marsden Hospital on children (aged less than 18), diagnosed to have acute Myeloid Leukaemia between January 2004 and June 2011.

Results A total of 72 patients were identified, of which 7 patients did not achieve remission following induction chemotherapy with ADE. 48 patients were appropriate for comparison, 22 patients 